\title{
Assisted reproductive technology and the risk of preterm birth among primiparas
}

\author{
Galit Levi Dunietz, M.A., M.P.H. ${ }^{a}$, Claudia Holzman, Ph.D., D.V.M. ${ }^{a}$, Patricia McKane, M.P.H., \\ D.V.M. ${ }^{b}$, Chenxi Li, Ph.D. ${ }^{a}$, Sheree L. Boulet, Dr.P.H. ${ }^{c}$, David Todem, Ph.D. ${ }^{a}$, Dmitry M. \\ Kissin, M.D., M.P.H. ${ }^{c}$, Glenn Copeland, M.B.A. ${ }^{d}$, Dana Bernson, M.P.H. ${ }^{e}$, William M. \\ Sappenfield, M.D. ${ }^{f}$, and Michael P. Diamond, M.D. ${ }^{g}$ \\ aDepartment of Epidemiology and Biostatistics, Michigan State University, East Lansing \\ bMichigan Department of Community Health, Maternal and Child Health Section, Lansing, \\ Michigan \\ 'Division of Reproductive Health at Centers for Disease Control and Prevention, Atlanta, Georgia \\ dMichigan Department of Community Health, Lansing, Michigan \\ eMassachusetts Department of Public Health, Boston, Massachusetts \\ fDepartment of Community and Family Health, University of South Florida, Tampa, Florida \\ 9Department of Obstetrics and Gynecology, Georgia Regents University, Augusta, Georgia
}

\begin{abstract}
Objective-To investigate the risk of preterm birth among liveborn singletons to primiparas who conceived with assisted reproductive technology (ART) using four mutually exclusive categories of infertility (female infertility only, male infertility only, female and male infertility, and unexplained infertility) and to examine preterm birth risk along the gestational age continuum.
\end{abstract}

Design—Retrospective cohort study.

Setting-Not applicable.

Patient(s)-Singletons born to primiparas who conceived with or without ART.

Intervention(s)-None.

Main Outcome Measure(s)—Preterm ( $<37$ weeks' gestation) and preterm/early term birth $<39$ weeks' gestation).

Result(s)-For the male infertility only, female infertility only, combined male and female infertility, and unexplained infertility groups, ART-conceived singletons were significantly more

Reprint requests: Galit Levi Dunietz, M.A., M.P.H., Michigan State University, Epidemiology and Biostatistics, 909 Fee Road, East Lansing, Michigan 48824 (galit@msu.edu).

G.L.D. has nothing to disclose. C.H. has nothing to disclose. P.M. has nothing to disclose. C.L. has nothing to disclose. S.L.B. has nothing to disclose. D.T. has nothing to disclose. D.M.K. has nothing to disclose.

The findings and conclusions in this report are those of the authors and do not necessarily represent the official position of the Centers for Disease Control and Prevention. 
likely than non-ART singletons to be born preterm: adjusted odds ratio (aOR) 1.24 (95\% CI, 1.13, 1.37), aOR 1.60 (95\% CI, 1.50, 1.70), aOR 1.49 (95\% CI, 1.35, 1.64), and aOR $1.26(1.12,1.43)$ respectively. Among infants whose mothers were diagnosed with infertility, the odds of preterm birth were highest between 28-30 weeks [female infertility only, aOR 1.95 (95\% CI, 1.59, 2.39); male and female infertility: 2.21 (95\% CI, 1.62, 3.00)] compared with infants in the general population. Within the ART population, singletons of couples with female infertility only were more likely to be born preterm than singletons born to couples with other infertility diagnoses.

Conclusion(s)-Among singleton births to primiparas, those conceived with ART had an increased risk for preterm birth, even when only the male partner had been diagnosed with infertility. The risk of preterm birth for ART-conceived infants whose mothers were diagnosed with infertility included the earliest deliveries.

\section{Keywords}

Assisted reproductive technology (ART); infertility; preterm birth

Assisted reproductive technology (ART) is a group of medical procedures for treating infertility in which both male and female gametes are handled outside the body to achieve conception. Since its introduction in 1978, ART has contributed to the birth of more than 5 million infants worldwide (1). In 2009, European registries reported that 109,239 infants were born after ART (2); in the United States, a total of 61,564 infants were born in 2010, representing, on average, $1.5 \%$ of its total births (3).

Although ART may help infertile couples achieve pregnancy, it also presents a public health challenge because of the reported associations with adverse birth outcomes such as preterm birth (PTB), low birth weight, and small for gestational age (GA) (4-6). Multifetal pregnancies are common in pregnancies achieved through ART (3) and are an important risk factor for PTB $(7,8)$. However, the association between ART and PTB is also observed in singletons $(9,10)$. Numerous studies have found a twofold risk increase for PTB in ARTconceived compared with non-ART conceived singleton pregnancies $(5,11)$. The explanation for this ARTPTB association remains unclear; the effect may be fully or partially confounded by other factors such as causes of the underlying infertility. One of the methodologic limitations of previous studies has been the composition of the control group, typically non-ART pregnancies.

The comparison of non-ART pregnancies with ART pregnancies does not provide an opportunity to disentangle effects associated with infertility versus effects of ART treatment. A recent meta-analysis (12) reported that a history of infertility among couples who conceived spontaneously is associated with a moderate risk for PTB, low birth weight, and small for GA. These results reinforce the problem of confounding by fertility-associated factors and demonstrate the need for alternative approaches to try and assess more specifically the effects of ART on adverse birth outcomes.

To elucidate the link of ART to adverse birth outcomes, we used data from the States Monitoring Assisted Reproductive Technology (SMART), a collaborative project of ART surveillance initiated by the Centers for Disease Control and Prevention (CDC) and the 
Massachusetts, Florida, and Michigan public health agencies. As previously described elsewhere (13), the SMART Collaborative was established to monitor and enhance ART surveillance within states and to study health outcomes among ART users. The SMART data set has been previously described. Briefly, it is a population-based data set of vital records of Massachusetts, Michigan, and Florida probabilistically linked to the National ART Surveillance System (NASS) data of all ART-associated deliveries (14).

The goals of our study were to [1] confirm previous investigations linking increased PTB risk with ART among singleton pregnancies, [2] extend this line of inquiry by considering whether excess PTB risk is confined to couples with female infertility, and [3] examine the ART-related excess risk of PTB across the GA continuum.

\section{MATERIALS AND METHODS}

We used a subset of SMART Collaborative data, with all singleton live births to primiparous women occurring in Massachusetts and Florida between the years 2000-2010 and Michigan 2000-2009 to examine GA at birth across study groups. We restricted the data set to primiparous women with singleton deliveries to avoid including multiple live births to the same woman, which would have resulted in correlated, nonindependent data. We excluded deliveries of women younger than 15 years of age and women older than 60 years because of the lack of comparable ART and non-ART groups, respectively.

Details on infertility diagnosis are part of the NASS data set and were used in the linked data file to create five mutually exclusive groups based on ART status and reason for infertility among ART patients: non-ART ( $\mathrm{n}=1,804,100)$, ART female infertility $(\mathrm{n}=9,891)$, ART male infertility $(\mathrm{n}=4,819)$, ART combined (male and female) infertility $(\mathrm{n}=3,688)$, and ART unexplained infertility $(n=2,930)$. The outcome of interest, GA at birth, was based on the clinical estimate obtained from birth files.

To avoid small cell sizes in contingency tables, our adjusted models included collapsed race/ ethnicity and education categories. Race/ethnicity categories of Hispanic and Asian/other were grouped into one, and similarly, both categories of "high school diploma or GED" and "less than high school education" were merged.

Initial analyses were conducted by comparing sociodemographic and pregnancy-relevant factors among the ART and non-ART groups with the purpose of identifying potential confounders. We used basic inferential statistical methods such as $t$ test, chi-square, and linear regression models. In the logistic regression models, odd ratios were calculated for PTB ( $<37$ weeks' gestation) and preterm/early term births ( $<39$ weeks' gestation). Binary logistic regression models compared PTB odds for each ART subgroup with that of nonART births. The male infertility only group was of particular interest as a means of examining ART outcomes in the absence of female infertility.

To gain more insight into the distribution of PTB risk, we further subclassified GA into five and six categories, with $>37$ and $>39$ weeks as the referent for PTB and preterm/early term birth, respectively. This strategy recognizes both uncertainties in GA dating and recent concerns for adverse outcomes even among early term births (37-38 weeks' gestation) (15). 
Recently, the American College of Obstetricians and Gynecologists suggested new definitions for full-term births as 239 to $<41$ gestation weeks (16).

Using multinomial logistic regression models, we next studied the odds of preterm $(<37$ weeks) versus term ( $>37$ weeks) birth along the continuum of preterm GA $(<28,28-30,31$ 33 , and 34-36 weeks) as well as the odds of preterm/early term ( $<39$ weeks) birth along the GA continuum of $<28,28-30,31-33,34-36$, and 37-38 weeks relative to $\geq 39$ weeks' gestation. Finally, we evaluated the association between the infertility diagnosis and PTB among ART births, using female infertility as the referent. This comparison was of particular interest as a means of assessing the likelihood of PTB according to infertility type among a subfertile population. Crude and adjusted odds ratios and $95 \%$ confidence intervals were calculated (SAS 9.3; SAS Institute); all adjusted models included maternal age, education, race, state, and year, which were derived from the birth certificate data. The study received approval from the institutional review boards of Massachusetts, Michigan, Florida, and the $\mathrm{CDC}$.

\section{RESULTS}

During the study period there were 21,328 (1.2\%) singleton deliveries to primiparous ART users and 1,804,100 (98.8\%) singleton deliveries to primiparous non-ART users (Table 1). The ART users were more likely to be older and non-Hispanic white, and to have a higher level of education compared with their non-ART counterparts ( $\mathrm{P}<.01$ for each comparison). The ART-conceived pregnancies were of shorter duration (mean GA 38.3-38.7 weeks) than the pregnancies not conceived through ART (mean GA 38.8 weeks; $P<.01$ ), and a higher percentage of ART-conceived pregnancies resulted in PTB (10\%-14\% among ART births versus $9 \%$ non-ART births; $P<.01)$.

In both crude and adjusted models for PTB <37 weeks' gestation, the odds were significantly higher among all ART groups compared with the non-ART referent group. Similarly, when 39 weeks' gestation was used as the cutoff to assess the risk of preterm and early term birth combined, all ART groups had significantly higher PTB odds compared with the non-ART deliveries (Table 2). After adjusting for maternal age, education, race, state, and year, we found that of all four infertility groups female infertility had the highest adjusted odds ratio (aOR) for PTB (aOR 1.60; 95\% CI, 1.50, 1.70). The adjusted odds ratios for couples with both female and male infertility and those with male infertility only were 1.49 (95\% CI, 1.35, 1.64) and 1.24 (95\% CI, 1.13, 1.37), respectively. Finally, the adjusted odds ratio for couples with unexplained infertility was 1.26 (95\% CI, 1.12, 1.43). Models using preterm/early term birth, defined as < 39 weeks' gestation, produced similar results with mostly attenuated aORs.

Next, we explored the odds of PTB along GA intervals $(<28,28-30,31-33,34-36$ weeks gestation with a referent category of 237 weeks' gestation) among infants conceived through ART by the type of parental infertility, as compared with infants not conceived through ART. The adjusted odds ratios for the female infertility group were 1.71 (95\% CI, 1.38, 2.13), 1.95 (95\% CI, 1.59, 2.39), 1.56 (95\% CI, 1.34, 1.81), and 1.57 (95\% CI, 1.46, 1.68) for birth before 28 weeks' gestation and between 28-30 weeks, 31-33 weeks, and 34- 36 
weeks versus 237 weeks, respectively (Table 3 ). A similar pattern was observed in the changes of GA-specific point estimates for male infertility only and combined infertility. We then used this approach to examine the odds of preterm and early term birth along the same GA intervals, with the exception that the later GA group was 37-38 weeks and $\geq 39$ weeks was the referent GA. The results were similar, with slightly higher PTB odds ratio estimates for all infertility groups (Table 3).

We used the Wald test to assess whether the PTB odds ratios displayed a heterogeneous pattern along the early GA continuum; only the combined infertility group showed a statistically significant heterogeneous pattern $(P=.02)$. For the outcome of preterm/early term birth ( $<39$ weeks' gestation), a heterogeneous pattern was detected for both female infertility and combined infertility groups $(P<.01)$. Supplemental Figure 1 (available online) provides a graphic display of the odds ratios for preterm/early term birth by GA.

For analyses within the ART population, the female infertility group served as the referent. We observed statistically significantly lower odds for PTB $<37$ weeks in both the male infertility group [aOR $0.78(95 \% \mathrm{CI}, 0.70,0.87)$ ] and the unexplained infertility group [aOR 0.79 (95\% CI, 0.69, 0.91)] (Table 4) when compared with the female infertility group. The models with PTB/early term birth defined as <39 weeks' gestation showed statistically significantly lower PTB odds in the male infertility and unexplained infertility groups relative to the female infertility group.

\section{DISCUSSION}

We found that, among singleton deliveries to primiparous women, the use of ART was associated with increased odds of PTB for couples with identified male infertility but without female infertility, when compared with births to non-ART users. These findings suggest that even in the absence of female infertility the use of ART increases the risk for PTB. In addition, we found that the odds of PTB were higher among couples with female infertility only or those with combined female and male infertility than among those of the male infertility group, thereby indicating that factors related to female infertility may further increase the risk of PTB among primiparous ART users. Indeed, comparisons within the ART population showed that singletons conceived via ART because of male infertility had a lower risk of PTB or preterm/early term birth compared with singletons born to mothers with female infertility.

When the preterm groups were further divided into subcategories, the increases risk associated with ART was not confined to late preterm and included the most vulnerable early preterm births, regardless of whether $>37$ weeks or $>39$ weeks was used as the referent category. When $>37$ weeks was used as the referent, variations in PTB odds ratios over the different GA categories were not statistically significant for the female and male infertility groups; however, they were statistically significant for the combined infertility group. When $>39$ weeks' gestation was used as the referent category for preterm/early term birth, the same heterogeneous pattern of odds ratios was observed in two groups: the female infertility and combined infertility groups. The observed variations in PTB odds across various GA thresholds may be due to pregnancy complications related to the infertility itself, the use of 
ART procedures, or their combination, as well as other unmeasured factors. As far as we know, our study is the first to report variability in PTB odds along the GA continuum and within specific ART subgroups involving female infertility or combined infertility diagnosis.

Biological explanations for our findings are likely heterogeneous and complex. One intriguing lead may come from the well-studied biomarker relaxin, a polypeptide hormone produced during pregnancy by the corpus luteum and decidua, which was linked to PTB because of its effect on collagen breakdown $(17,18)$. In animal models, it was shown that the involvement of relaxin in cervical collagenolysis decreased cervical resistance and led to consequent cervical ripening (19). Compared with naturally conceived pregnancies, those conceived through ovulation induction had higher circulating levels of relaxin not only in the first trimester (20) but also in the second and third trimesters (21). Finally, higher relaxin levels observed at the third trimester were significantly associated with PTB (18). Other suggested explanations for the higher PTB risk among ART singletons include placental abnormalities $(11,22)$, a co-twin fetal reabsorption (23), transfer of fresh versus frozen embryos (24), and use of donor versus autologous oocytes (25).

Our study has several strengths. First, previous studies compared the PTB risk between pregnancies achieved with and without the use of ART, but we were able to divide the ART population into four distinct groups with respect to their infertility diagnosis, thereby providing specific PTB risks by infertility diagnosis. Second, the SMART Collaborative database is comprehensive and unique as it includes numerous variables related to both exposures and outcomes. The ART variables are of particularly high quality because they are collected via a federally mandated reporting system with approximately $7 \%-10 \%$ of reporting clinics randomly selected for validation each year. Consequently, the data provide us with the opportunity to refine previous study questions and conduct novel analyses among distinct subgroups of the population. Finally, by linking multiple years of data, the sample size and thus statistical power are increased.

There are also limitations to consider. First, the probabilistic linkage method of birth files and NASS data resulted in a high success rate, but it is not free of matching errors (14). Second, some women in the unexposed group, the non-ART group, may have been exposed to hormones, through non-IVF ovulation induction or ovarian stimulation protocols. In addition a small percentage of couples in the non-ART group may have experienced subfertility similar to the ART group but continued to attempt conception without ART and were then successful. This would likely have the effect of attenuating the odds ratios of PTB related to infertility. Third, the female infertility group is not homogeneous with respect to the infertility diagnosis; rather, it is a collection of different conditions related to several mechanical or hormonal dysfunctions. Similarly, the infertility groups are heterogeneous with respect to the specifics of ART procedures. Therefore, the PTB risk may differ based on the underlying condition of female infertility diagnosis and on ART treatments. Fourth, the GA at birth was based on birth certificate data for both non-ART and ART pregnancies. It is possible that birth file GA estimates are better informed (more accurate) among ART pregnancies when embryo transfer dates are known. Finally, the large size of our data set allows detection of small differences that are statistically significant but may not be of clinical importance. 


\section{CONCLUSIONS}

The risk for PTB for ART-conceived pregnancies, even in the absence of female infertility, is higher than for pregnancies in the general population. A female infertility diagnosis, as opposed to male infertility alone, may be associated with a greater increase in the likelihood of PTB in ART births. The increased risk of PTB associated with ART is not confined to late PTB, and among the ART subset with female infertility or combined male and female infertility, the risk is inversely related to GA at birth.

\section{Supplementary Material}

Refer to Web version on PubMed Central for supplementary material.

\section{Acknowledgments}

The authors thank Ajay Yesupriya and Yujia Zhang for technical assistance.

G.C. has received a grant from the Centers for Disease Control and Prevention. D.B. has nothing to disclose. W.M.S. has received a grant from the Florida Department of Public Health. M.P.D. is a stockholder in and on the board of directors of Advanced Reproductive Care, and has received a grant from the NIH/NICHD Cooperative Reproductive Medicine Network.

This work was supported in part by a T32 grant from the Eunice Kennedy Shriver National Institute of Child Health and Human Development (T32-HD046377).

\section{References}

1. Bauquis, C. The world's number of IVF and ICSI babies has now reached a calculated total of 5 million. European Society of Human Reproduction and Embyology. 2012. Available at: http:// www.eshre.eu/ESHRE/English/Press-Room/Press-Releases/Press-releases-2012/5-million-babies/ page.aspx/1606

2. Ferraretti AP, Goossens V, Kupka M, Bhattacharya S, de Mouzon J, Castilla JA, et al. Assisted reproductive technology in Europe, 2009: results generated from European registers by ESHRE. Hum Reprod. 2013; 28:2318-31. [PubMed: 23842560]

3. Sunderam S, Kissin DM, Crawford S, Anderson JE, Folger SG, Jamieson DJ, et al. Assisted reproductive technology surveillance-United States, 2010. MMWR Surveill Summ. 2013; 62:124. [PubMed: 24304902]

4. D’Angelo DV, Whitehead N, Helms K, Barfield W, Ahluwalia IB. Birth outcomes of intended pregnancies among women who used assisted reproductive technology, ovulation stimulation, or no treatment. Fertil Steril. 2011; 96:314-20.e2. [PubMed: 21718990]

5. McGovern PG, Llorens AJ, Skurnick JH, Weiss G, Goldsmith LT. Increased risk of preterm birth in singleton pregnancies resulting from in vitro fertilization-embryo transfer or gamete intrafallopian transfer: a meta-analysis. Fertil Steril. 2004; 82:1514-20. [PubMed: 15589852]

6. Schieve LA, Meikle SF, Ferre C, Peterson HB, Jeng G, Wilcox LS. Low and very low birth weight in infants conceived with use of assisted reproductive technology. N Engl J Med. 2002; 346:731-7. [PubMed: 11882728]

7. McDonald SD, Han Z, Mulla S, Ohlsson A, Beyene J, Murphy KE. Preterm birth and low birth weight among in vitro fertilization twins: a systematic review and meta-analyses. Eur J Obstet Gynecol Reprod Biol. 2010; 148:105-13. [PubMed: 19833428]

8. Murray SR, Norman JE. Multiple pregnancies following assisted reproductive technologies-a happy consequence or double trouble? Semin Fetal Neonatal Med. 2014; 19:222-7. [PubMed: 24685368]

9. Hayashi M, Nakai A, Satoh S, Matsuda Y. Adverse obstetric and perinatal outcomes of singleton pregnancies may be related to maternal factors associated with infertility rather than the type of 
assisted reproductive technology procedure used. Fertil Steril. 2012; 98:922-8. [PubMed: 22763098]

10. Tepper NK, Farr SL, Cohen BB, Nannini A, Zhang Z, Anderson JE, et al. Singleton preterm birth: risk factors and association with assisted reproductive technology. Matern Child Health J. 2012; 16:807-13. [PubMed: 21516300]

11. Jackson RA, Gibson KA, Wu YW, Croughan MS. Perinatal outcomes in singletons following in vitro fertilization: a meta-analysis. Obstet Gynecol. 2004; 103:551-63. [PubMed: 14990421]

12. Messerlian C, Maclagan L, Basso O. Infertility and the risk of adverse pregnancy outcomes: a systematic review and meta-analysis. Hum Reprod. 2013; 28:125-37. [PubMed: 23042798]

13. Mneimneh AS, Boulet SL, Sunderam S, Zhang Y, Jamieson DJ, Crawford S, et al. States Monitoring Assisted Reproductive Technology (SMART) Collaborative: data collection, linkage, dissemination, and use. J Womens Health (Larchmt). 2013; 22:571-7. [PubMed: 23829183]

14. Zhang Y, Cohen B, Macaluso M, Zhang Z, Durant T, Nannini A. Probabilistic linkage of assisted reproductive technology information with vital records, Massachusetts 1997-2000. Matern Child Health J. 2012; 16:1703-8. [PubMed: 21909704]

15. Sengupta S, Carrion V, Shelton J, Wynn RJ, Ryan RM, Singhal K, et al. Adverse neonatal outcomes associated with early-term birth. JAMA Pediatr. 2013; 167:1053-9. [PubMed: 24080985]

16. ACOG Committee Opinion No 579: Definition of term pregnancy. Obstet Gynecol. 2013; 122:1139-40. [PubMed: 24150030]

17. Bryant-Greenwood GD, Yamamoto SY, Lowndes KM, Webster LE, Parg SS, Amano A, et al. Human decidual relaxin and preterm birth. Ann N Y Acad Sci. 2005; 1041:338-44. [PubMed: 15956731]

18. Petersen LK, Skajaa K, Uldbjerg N. Serum relaxin as a potential marker for preterm labour. Br J Obstet Gynaecol. 1992; 99:292-5. [PubMed: 1581273]

19. Luque EH, Munoz de Toro MM, Ramos JG, Rodriguez HA, Sherwood OD. Role of relaxin and estrogen in the control of eosinophilic invasion and collagen remodeling in rat cervical tissue at term. Biol Reprod. 1998; 59:795-800. [PubMed: 9746727]

20. Weiss G, Goldsmith LT, Sachdev R, Von Hagen S, Lederer K. Elevated first-trimester serum relaxin concentrations in pregnant women following ovarian stimulation predict prematurity risk and preterm delivery. Obstet Gynecol. 1993; 82:821-8. [PubMed: 8414331]

21. Mushayandebvu TI, Goldsmith LT, Von Hagen S, Santoro N, Thurston D, Weiss G. Elevated maternal serum relaxin concentrations throughout pregnancy in singleton gestations after superovulation. Obstet Gynecol. 1998; 92:17-20. [PubMed: 9649085]

22. Schieve LA, Cohen B, Nannini A, Ferre C, Reynolds MA, Zhang Z, et al. A population-based study of maternal and perinatal outcomes associated with assisted reproductive technology in Massachusetts. Matern Child Health J. 2007; 11:517-25. [PubMed: 17345154]

23. Pinborg A, Lidegaard O, la Cour Freiesleben N, Andersen AN. Consequences of vanishing twins in IVF/ICSI pregnancies. Hum Reprod. 2005; 20:2821-9. [PubMed: 15979998]

24. Maheshwari A, Pandey S, Shetty A, Hamilton M, Bhattacharya S. Obstetric and perinatal outcomes in singleton pregnancies resulting from the transfer of frozen thawed versus fresh embryos generated through in vitro fertilization treatment: a systematic review and meta-analysis. Fertil Steril. 2012; 98:368-77. e1-9. [PubMed: 22698643]

25. Malchau SS, Loft A, Larsen EC, Aaris Henningsen AK, Rasmussen S, Andersen AN, et al. Perinatal outcomes in 375 children born after oocyte donation: a Danish national cohort study. Fertil Steril. 2013; 99:1637-43. [PubMed: 23465822] 


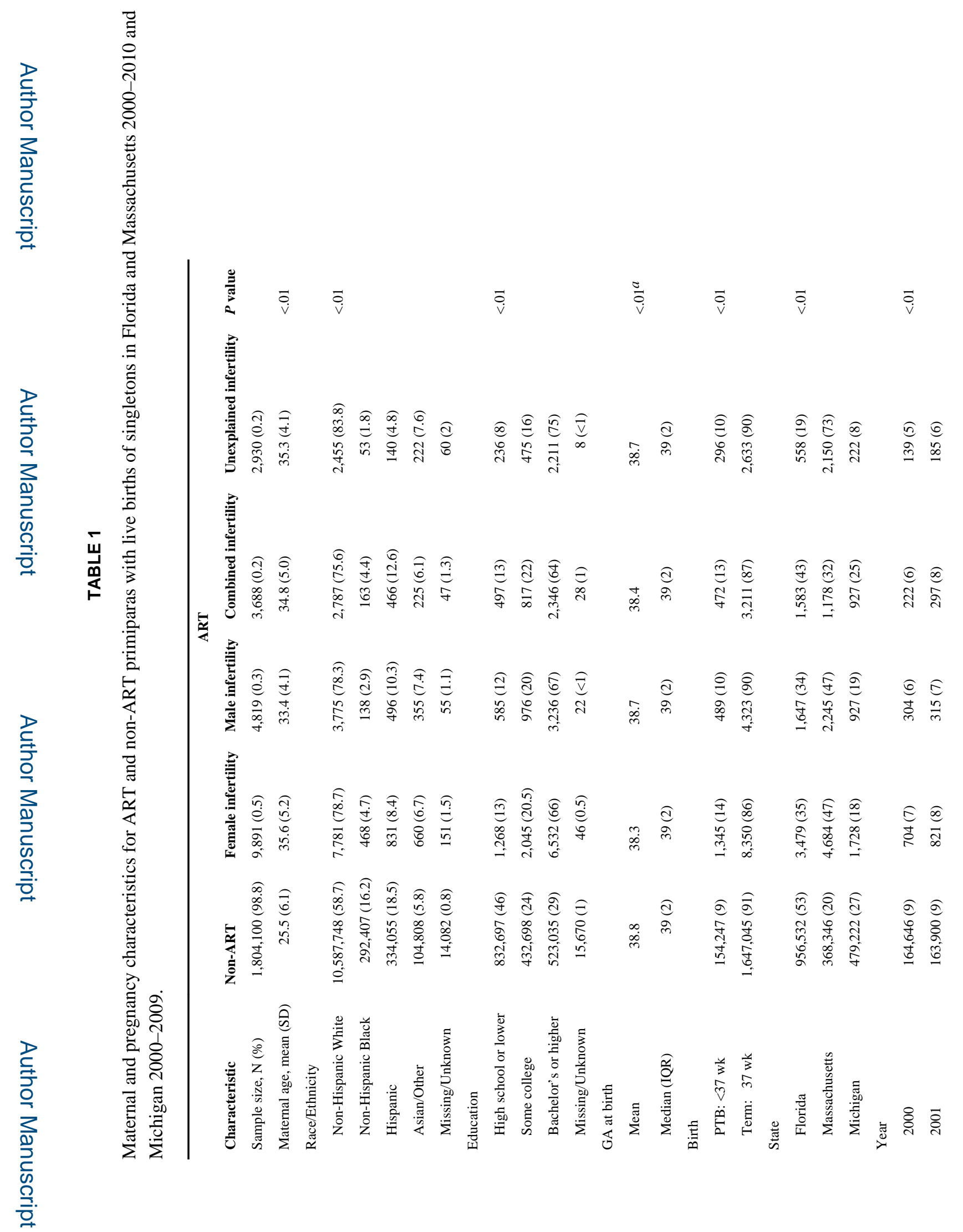

Fertil Steril. Author manuscript; available in PMC 2016 April 01. 


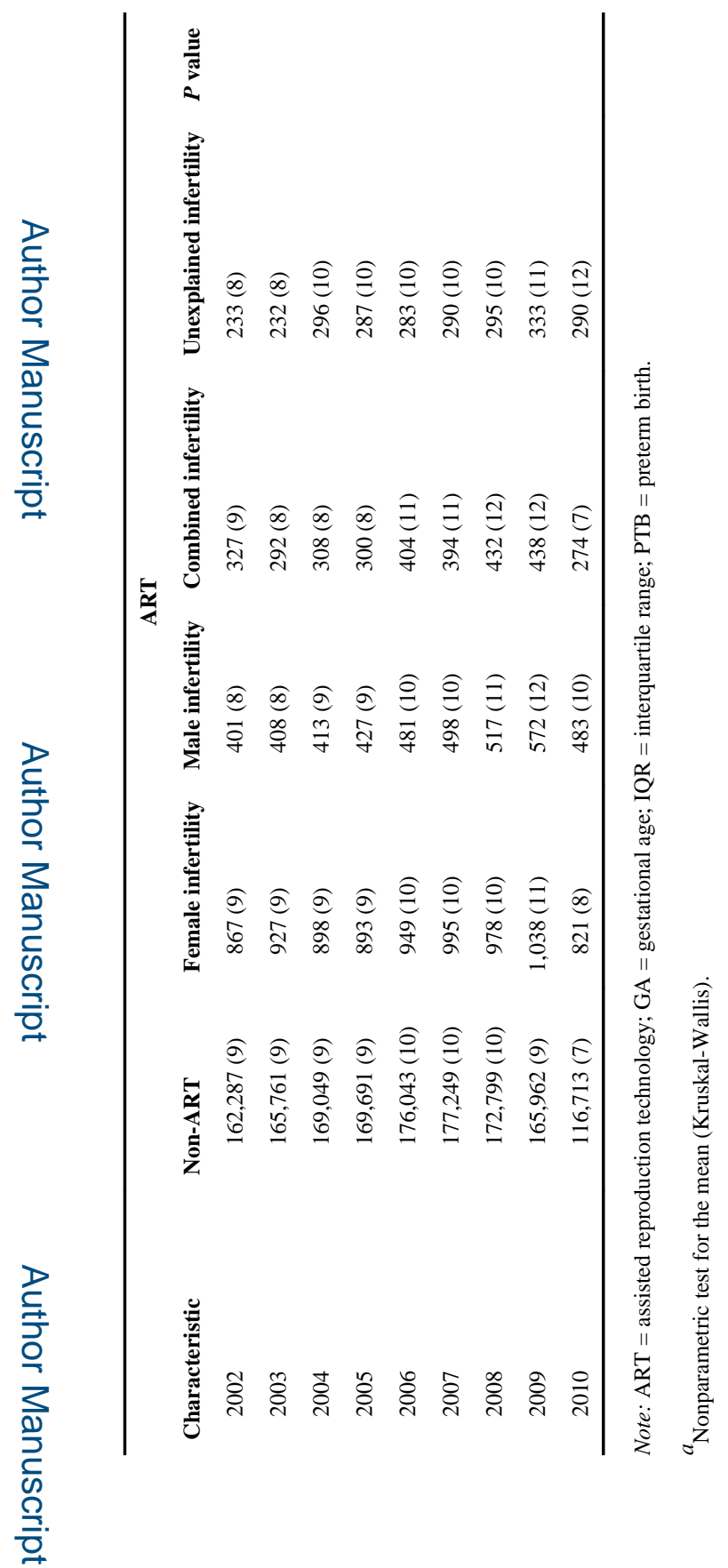

Fertil Steril. Author manuscript; available in PMC 2016 April 01. 


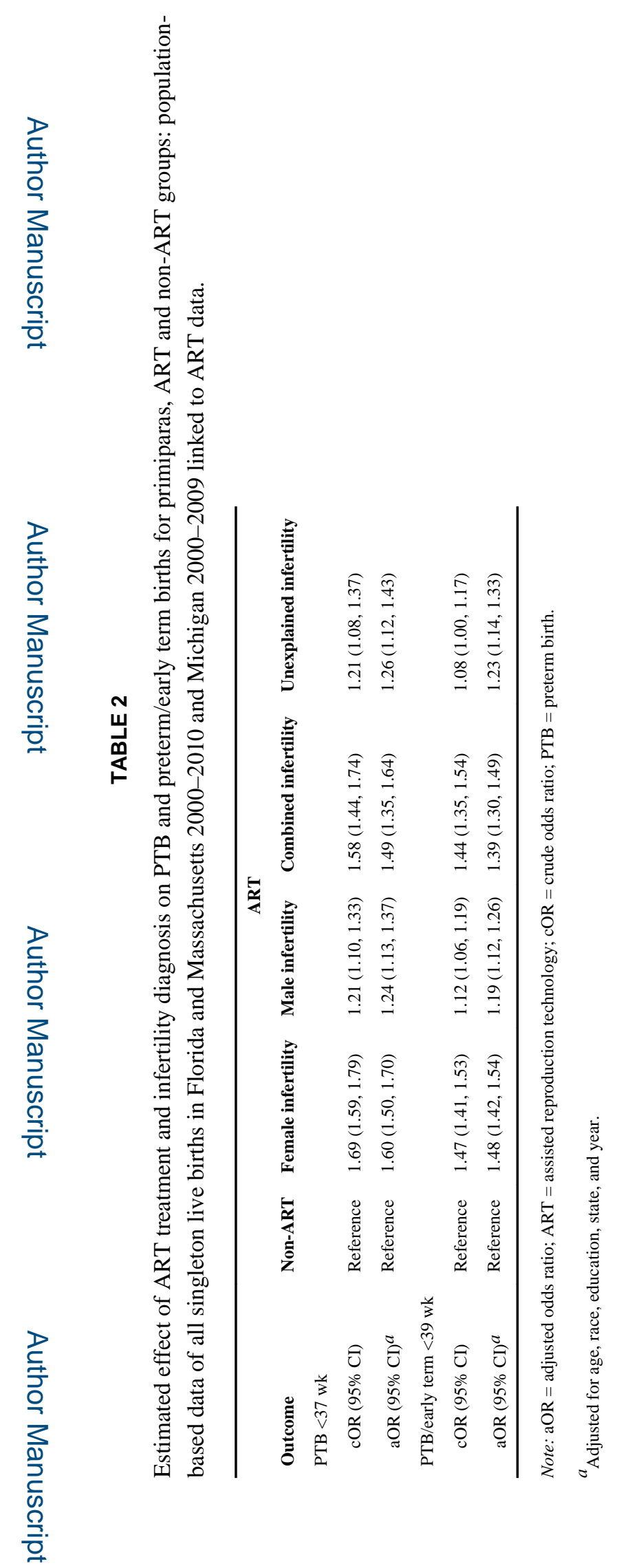

Fertil Steril. Author manuscript; available in PMC 2016 April 01. 
Dunietz et al.

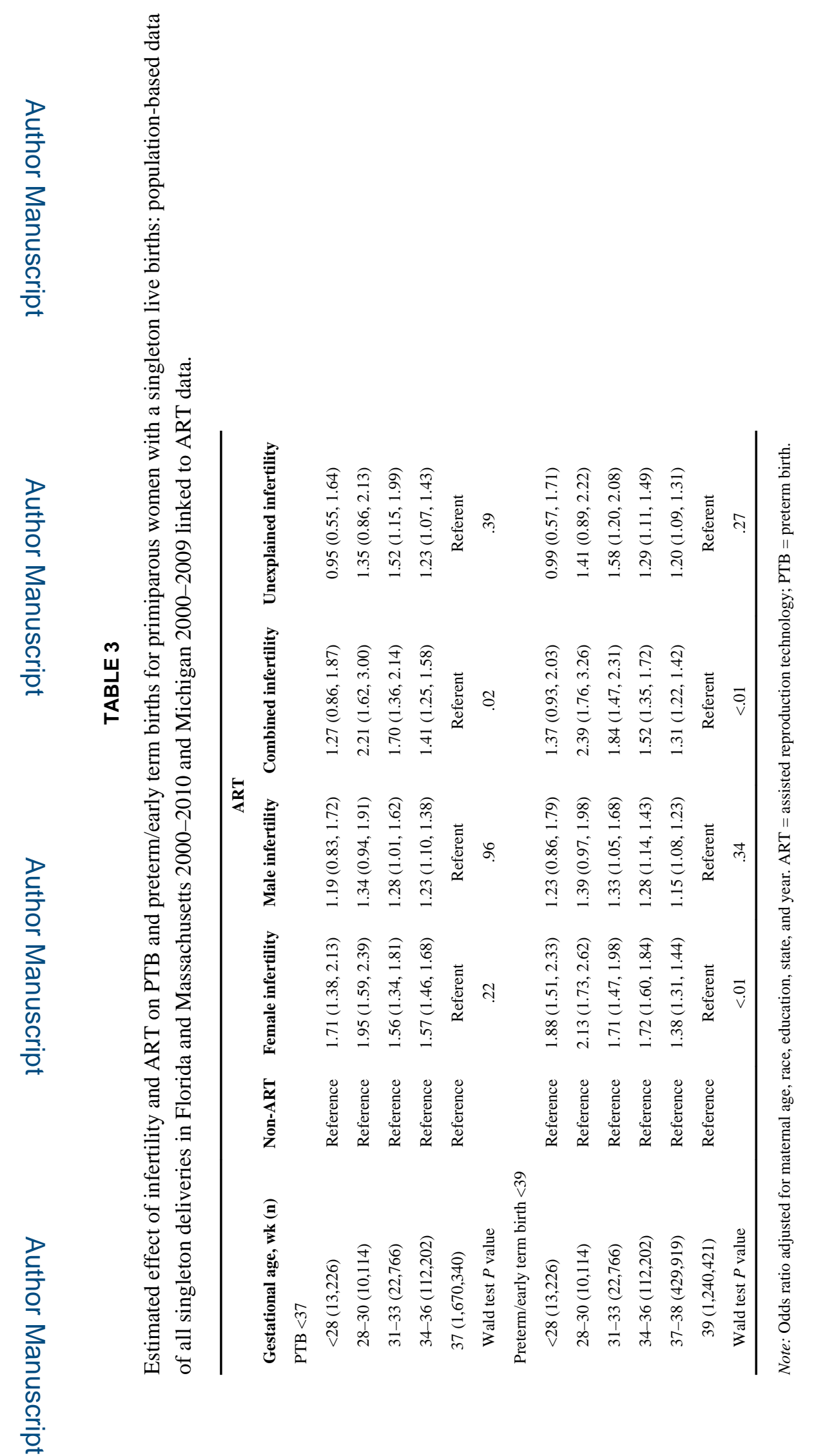

Fertil Steril. Author manuscript; available in PMC 2016 April 01. 


\section{TABLE 4}

Estimated effect of infertility on PTB and preterm/early term deliveries for primiparous, ART-users: population-based data of all singleton live births in Florida and Massachusetts 2000-2010 and Michigan 2000-2009 linked to ART data.

\begin{tabular}{lcccc}
\hline & & \multicolumn{3}{c}{ Infertility } \\
Outcome & Female & Male & Combined & Unexplained \\
Sample size $(\mathrm{n})$ & 9,891 & 4,819 & 3,688 & 2,930 \\
$\begin{array}{l}\text { PTB }<37 \mathrm{wk} \\
\text { cOR }(95 \% \mathrm{CI})\end{array}$ & & & & \\
aOR $(95 \% \mathrm{CI})^{a}$ & Reference & $0.72(0.64,0.80)$ & $0.94(0.84,1.05)$ & $0.72(0.63,0.82)$ \\
$\begin{array}{l}\text { Preterm/early term birth }<39 \mathrm{wk} \\
\text { cOR }(95 \% \mathrm{CI})\end{array}$ & Reference & $0.78(0.70,0.87)$ & $0.93(0.83,1.05)$ & $0.79(0.69,0.91)$ \\
$\mathrm{aOR}(95 \% \mathrm{CI})^{a}$ & Reference & $0.76(0.71,0.82)$ & $0.98(0.91,1.06)$ & $0.74(0.68,0.81)$ \\
\hline
\end{tabular}

Note: $\mathrm{aOR}=$ adjusted odds ratio; $\mathrm{ART}=$ assisted reproduction technology; $\mathrm{cOR}=$ crude odds ratio; $\mathrm{PTB}=$ preterm birth.

${ }^{a}$ Adjusted for age, race, education, state, and year. 